\title{
Accretion Processes in Astrophysics - II (Concluding Address)
}

\section{Franco Giovannelli*}

INAF - Istituto di Astrofisica e Planetologia Spaziali, Via del Fosso del Cavaliere, 100, 00133

Roma, Italy

E-mail: Eranco.qiovannellidiaps.inaf.it

Before I officially conclude this workshop - and far be it from me to attempt more conclusive remarks than those effected by Nikolai Shakura, Paul Mason, Dmitry Bisikalo, and Janusz Ziółkowski - I would like to comment on a few highlights coming from our very fruitful week of discussions about Accretion Processes in Cosmic Sources - II. I make no pretension of completeness in these brief remarks. For a clear short summary about my scientific route of course immersed in that larger of my life I invite readers to see the concluding address of the first edition of this series of workshops (Giovannelli, 2016). I hope that this could be useful for the readers, especially if young.

Accretion Processes in Cosmic Sources - II - APCS2018

3-8 September 2018

Saint Petersburg, Russian Federation

\footnotetext{
* Speaker.
} 


\section{Antecedent fact}

In this era - very troubled by major events, such as the overpopulation of the planet, the unstoppable migratory flows, due to the looting of the poorest countries, the run-up to nuclear weapons in the false illusion that peace can be guaranteed by weapons, poisoning apparently unstoppable of the planet - humanity is going along a wrong path.

So where are we going and for what purpose?

All human efforts since long time are inspired by the attempt to answer these fundamental questions:

- Where do we come from?

- Where are we going?

- Why we are here?

Indeed, Lev Nikolayevich Tolstoy in his novel "Anna Karenina" describes the conversation between Anna and Levin, in which she says: Who are we? Where do we come from? Where do we go?

The same questions were posed by Paul Gauguin in 1897 when he painted his famous picture, preserved in the Museum of Fine Arts in Boston and shown in Figure 1. The figures present humanity in the different states of life, from infancy to old age, in a mysterious and indeterminate nature.

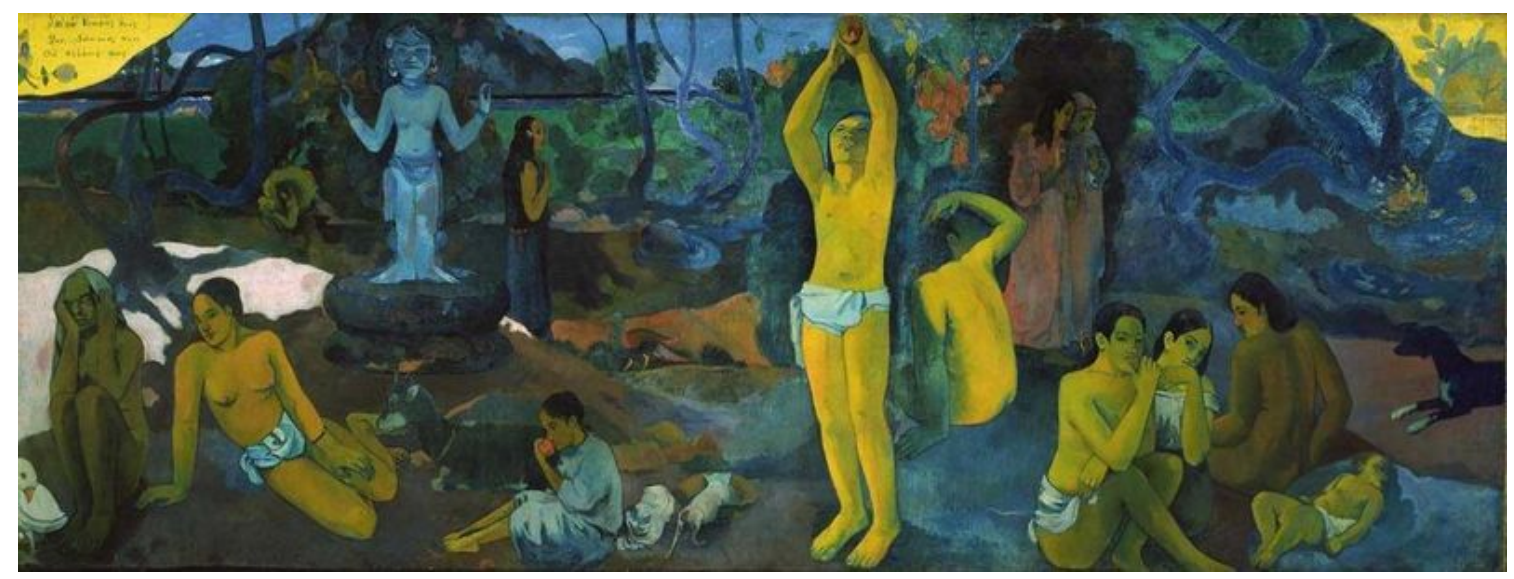

Figure 1: Where do we come from? Who we are? Where do we go? (Paul Gauguin, 1897, Museum of Fine Arts, Boston, Ma, USA).

Indeed, the canvas has a horizontal development with a reading path that goes right to left. Along this direction, Gauguin has a series of figures that essentially repeats the "Allegories of the Ages of Life". From the newborn in the right corner we reach the dark woman on the left passing through the various seasons of life. The woman at the center, who almost divides the picture into two, symbolizes the moment of life in which the fruits are gathered, an obvious allegory of the moment of procreation. The old woman on the far left, already present in other compositions by 
Gauguin, in her fetal position with her hands next to her face, does not really symbolize only old age but above all the fear of death.

But extraordinary in this context is above all the setting. The path of life takes place in a garden that really knows of Eden. As if to say that, according to Gauguin, after all, life and reality are not bad, were it not for the anguish of not knowing for sure what it is all about.

With this picture the sense of uneasiness and instability, typical of the artist and man Gauguin, appears at the end as an endless journey, because it is aimed at goals that are not of this world. And so his escape from the West to the paradises of the South Seas, after all, is nothing but the metaphor, not figured but real, of the perennial but inexhaustible research of the final landing of our serenity.

To try to answer these questions, scientists must work within the field of their competence without forgetting the scientific rigor, accompanied by an unyielding ethic that can make their work more humanized and above all always the use of wisdom, avoiding to proceed with blinkers.

\section{Search for exoplanets}

The presence of numerous exoplanets in the vicinity of solar system - within a distance of $\sim$ $0.9 \mathrm{kpc}$, which is the range accessible by the Kepler mission - plays an important role in speculating about the possible number of such exoplanets within the whole habitable zone of our galaxy.

As discussed in the paper by Giovannelli \& Sabau-Graziati (2018), planets around other stars are the rule rather than the exception, and there are likely hundreds of billions of exoplanets in the Milky Way alone (Maruyama, Ebisuzaki \& Kurokawa, 2018). Therefore, it is evident that the probability of finding numerous habitable planets becomes very high. Next generation instruments ground- and space-based will provide valuable information about this intriguing problem.

For life-forms like us, the most important feature of Earth is its habitability. Understanding habitability and using that knowledge to locate the nearest habitable planet may be crucial for our survival as a species. During the past decade, expectations that the universe could be filled with habitable planets have been bolstered by the increasingly large overlap between terrestrial environments known to harbor life and the variety of environments on newly detected rocky exoplanets. The inhabited and uninhabited regions on Earth tell us that temperature and the presence of water are the main constraints that can be used in a habitability classification scheme for rocky planets. Lineweaver \& Chopra (2012) compiled and reviewed the recent exoplanet detections suggesting that the fraction of stars with planets is $\sim 100 \%$, and that the fraction with rocky planets may be comparably large.

However, we have interesting news about the presence of water in the universe. We knew that all the water found on Earth, has been transported by small bodies such as comets and asteroids. On the contrary, the work "The ancient heritage of water ice in the solar system" (Cleeves et al., 2014) has carried the knowledge one step further. It is understood that the water now present in Earth's oceans, and is present in other solar system bodies, has remained virtually unchanged with respect to that in the interstellar medium. This means that this water has not changed during the process of planet formation. This allows us to understand that the initial conditions that have favored the emergence of life are not unique, i.e. not dependent on the unique characteristics of our solar system. They can, however, be common in space. 
By using the European Southern Observatory's HARPS - a high precision instrument fitted to the 3.6-m telescope at the La Silla Observatory in Chile - 102 red dwarf stars neighbouring the sun over a period of six years have been studied. Red dwarfs are smaller and cooler than the Sun, however it's been found that $40 \%$ of red dwarf stars may have Earth-sized planets orbiting them that have the right conditions for life. New observations with Harps mean that about $40 \%$ of all red dwarf stars have a super-Earth orbiting in the habitable zone where liquid water can exist on the surface of the planet. Because red dwarfs are so common - there are about 160 billion of them in the Milky Way - this leads us to the astonishing result that there are tens of billions of these planets in our galaxy alone (Bonfils et al., 2013).

Therefore, planets around other stars are the rule rather than the exception, and there are likely hundreds of billions of exoplanets in the Milky Way alone. NASA's Kepler space telescope has found more than 2,400 alien worlds, including a new haul of 95 planets announced on Feb. 15, 2018.

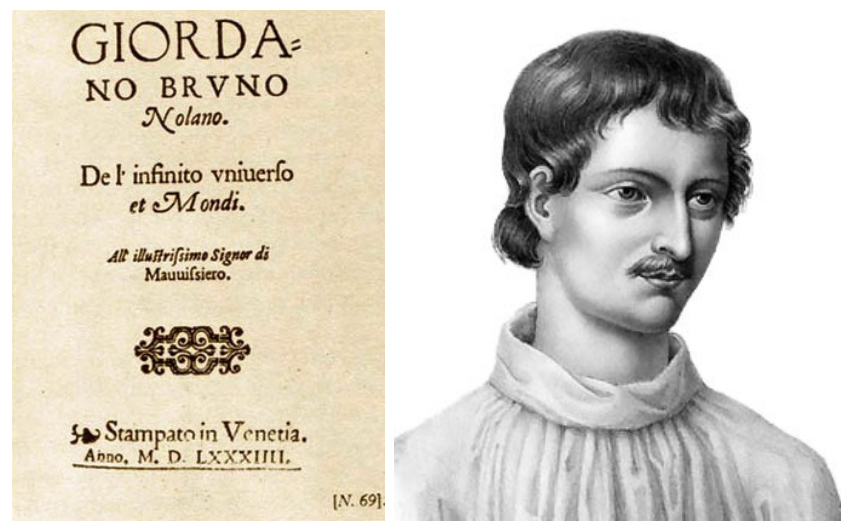

Figure 2: Left panel: Frontispiece of the original publication of De l'infinito, universo et mondi (Giordano Bruno, 1584). Right panel: Giordano Bruno (Rixner \& Siber, 1824).

However, we are going to study 50,000 Clusters of Galaxies (Thomas Boller, 2017), and we have millions more. We know the lower limit of the extrapolated number of Earth-size planets in the habitable zone of our Galaxy. Thus a natural extrapolation can be reasonably thought. Thus, we can dare to say that we are approaching to the philosophical results obtained by two great free thinkers:

- Siddhartha Gautama also known as Shakyamuni (the sage of Shaka - between the VI and V century B.C.) who exposes a grandiose vision of the universe: through the concept of "major system of worlds", a concept on huge scale that implies both the existence of countless galaxies and the possibility of sentient life on other planets other than our own (from the Lotus Sutra - the central text of Mahayana Buddhism).

- About 2000 years later, Giordano Bruno (Nola 1548 - Roma 17th February 1600) who was burned alive in Campo dei Fiori by the "Saint Inquisition" because of his thought - summarized in De l'infinito, universo e mondi (Giordano Bruno, 1584) (see Fig. 2) - that produced the same conclusions of Siddharta: The Universe is infinite and is populated by a myriad of worlds. Moreover he was saying that "Whether we like it or not, we are the cause of 
ourselves. Being born in this world, we fall into the illusion of the senses: we believe in what appears. We ignore that we are blind and deaf. Then the fear attacks us and we forget that we are "divine". We can change the course of events".

These philosophical lucubrations were not exactly in agreement with the position of the Roman (Catholic) Church!

Of course we must wait scientific confirmation for the "alien life". We must wait even more for the discovery of "intelligent life". But, the number of discovered planets is growing very fast. Thus, we can reasonably affirm that the Universe is full of life, hoping to avoid to be burned alive like Giordano Bruno.

An intriguing question about the probability of finding a number of civilization in the Galaxy arises. It is now evident that Drake's formula (Drake, 1962) must be object of a robust revision.

It is important for me to close this section with the beautiful words full of hope of William Borucki - principal investigator for NASA's Kepler mission: If we find lots of planets like ours... we'll know it's likely that we aren't alone, and that someday we might be able to join other intelligent life in the universe.

\section{Accretion: A general process in nature}

With the advent of ALMA (The Atacama Large Millimeter/submillimeter Array) - a worldwide collaboration (ESO/NAOJ/NRAO) - (ALMA Science Advisory Committee, 2015; Carpenter et al., 2018) unbelievable advancement have been obtained in many fields of astrophysics, and in particular in that of protoplanetary science.

The theory explaining the formation of protoplanetary disks is in excellent agreement with the experimental results, as shown in Fig. 3 (Antoniucci, 2018, this wokshop). These new results represent an enormous step forward in the understanding of how protoplanetary disks develop and how planets form.
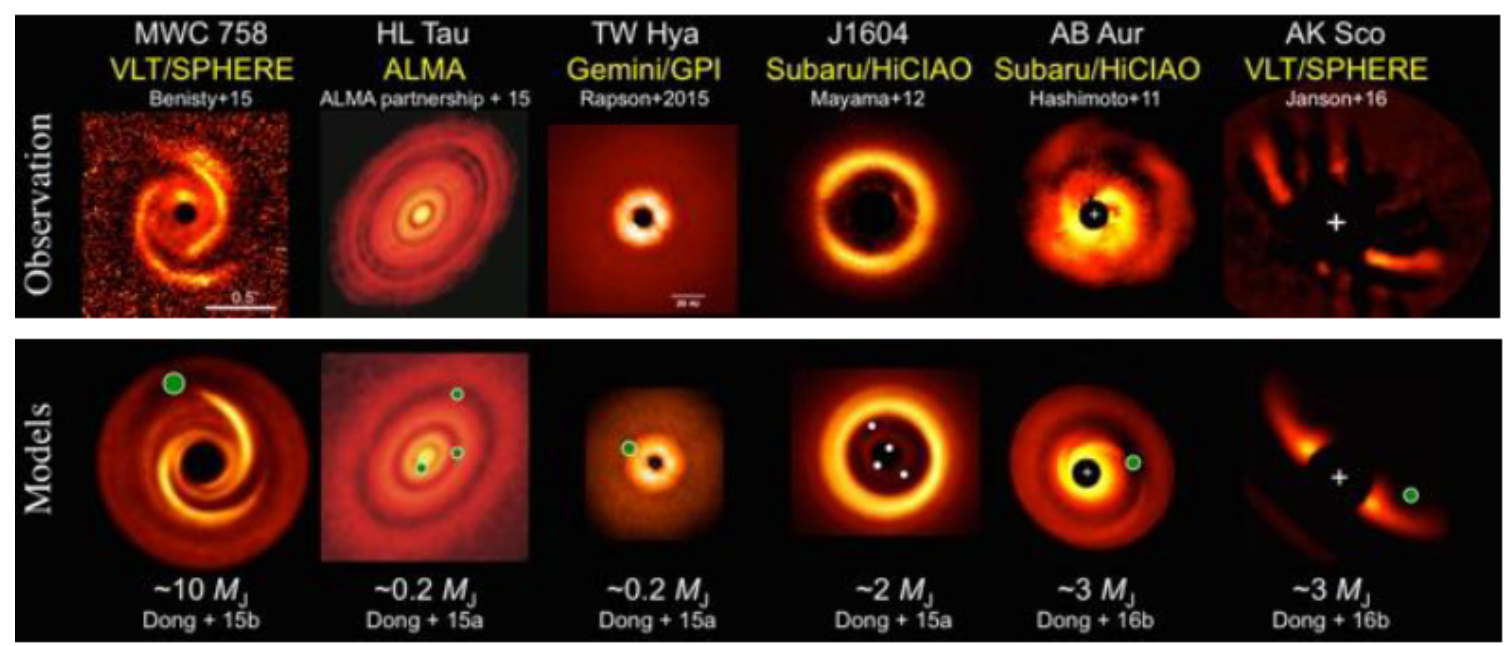

Figure 3: Upper panel: Observations of several systems. Lower panel: Models for the corresponding systems (courtesy of Simone Antoniucci, 2018). 
Bruno Luigi Martino with an amusing joke has shown that really the accretion is a universal process as shown in Fig. 4.

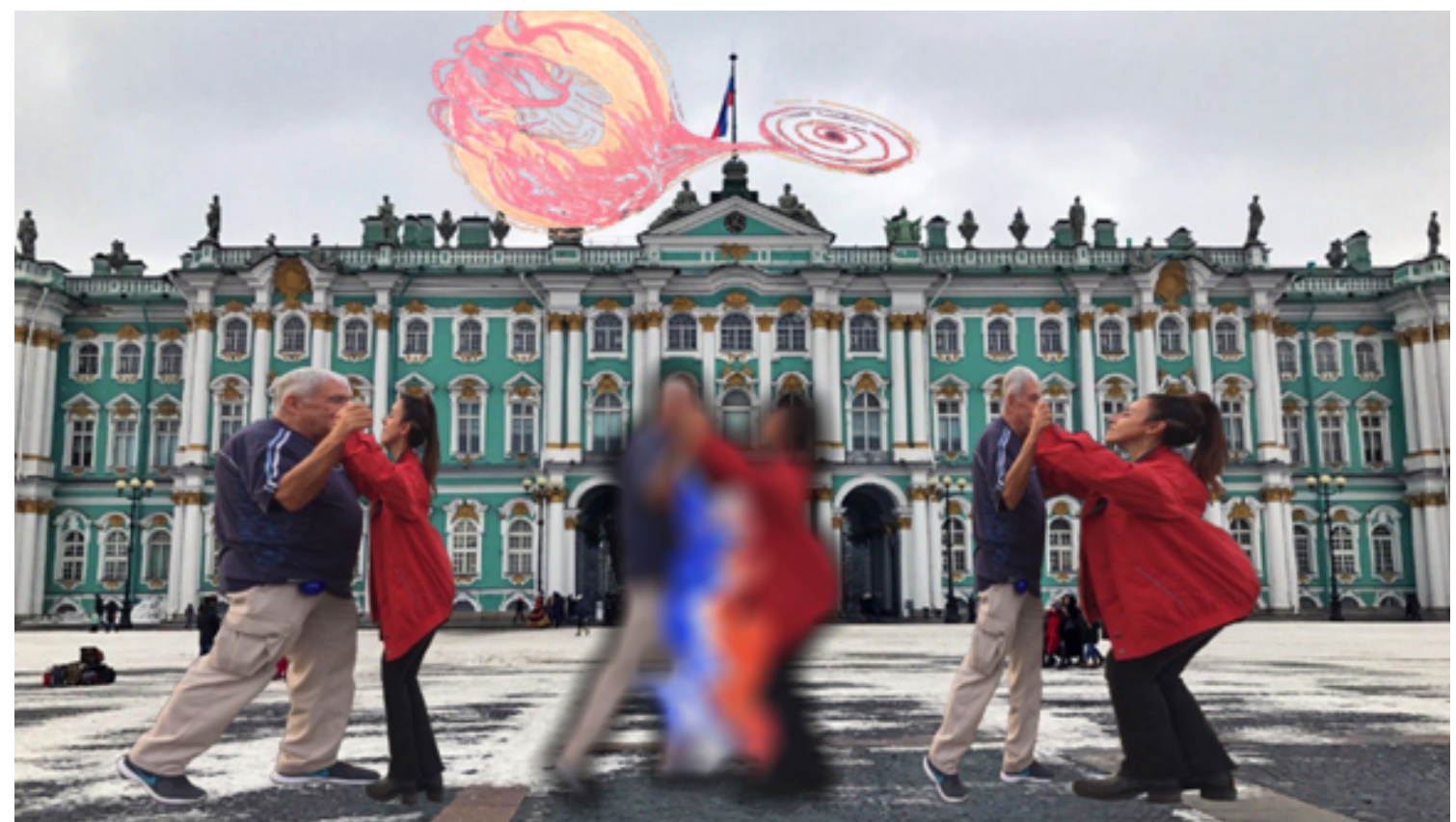

Figure 4: Accretion dance on front of the Winter Palace (credit: Bruno Luigi Martino, 2018).

\section{The Vulcano Theorem}

During this fruitful workshop, we hope to have demonstrated once more the "Vulcano Theorem" enunciated in 1984 in my concluding address of the first historical workshop on "Multifrequency Behaviour of Galactic Accreting Sources" (Giovannelli, 1985): It is possible to develop science seriously even if smiling.

In this workshop, the presence of women has been particularly pleasant and intentional as well as the presence of many young colleagues, some of them still PhD students.

This is the age of the youth. Young people do not depend on anyone or draw strength from others. The courage of young people is unparalleled. It fears nothing. The courage of youth is boundless, is the strength to never give up (Daisaku Ikeda, 2001a).

And I would like to remind one famous sentence of Leonardo Da Vinci: Tristo è lo discepolo che non supera lo maestro suo!, that in English is Grim is the disciple who does not exceed his master!

And finally, I would like to conclude with few wonderful words of Dr Daisaku Ikeda (2001b), president of the Soka Gakkai International (SGI), and reported in the booklet entitled For Today and Tomorrow as the thought of the 30th of May: "The one who has many friends has greater opportunities for growth. In this way, one both makes society a better place, and lives happier and more satisfied. In all cases, human relations, the inter-personal interaction and communication are of vital importance. We must establish and nurture friendship and contacts with many people, 
both in our environment, and in society in general. In this manner our life will open up and will flourish".

We could go back to early childhood when we were as the "Little Prince", who says that One sees clearly only with the heart. What is essential is invisible to the eye (from The Little Prince by Antoine de Saint Exupéry, 1943).

The search for the essential is of extreme interest to a large number of men of great learning. These are in agreement with Paul Salahuddin Armstrong, who said in his 2014 talk "Human Family; Past, Present and Future", at the "New Humanity Movement-Event" (Paul Salahuddin Armstrong, 2014): Today we travel the world, making connections, doing business, and building relationships in person or online with fellow members of our Human Family from all parts of the Earth. We are becoming more conscious that what happens in one place affects people everywhere. We are not alone... We are not isolated... Only through building bridges of Love and Understanding can we ensure the well-being of everyone in our Human Family.

The search for the essential is so important that even famous, noble-minded scientists try to attempt the difficult way of the possible convergence of science and life in its more sublime meaning. For instance, Pier Luigi Luisi founded in 1985 the International Week of Cortona "Science and the Wholeness of Life", dedicated to the integration of Scientific Disciplines and Humanities. Later he published the book "The emergence of Life. From Chemical Origins to Synthetic Biology (Luisi, 2006) in which he resumed the consecutive stages from prebiotic chemistry to synthetic biology, uniquely combining both approaches. Indeed, the origin of life from inanimate matter has been the focus of much research for decades, both experimentally and philosophically. Friedrich Rolle, a German philosopher and biologist, wrote "The general reasons for this assumption are so categorical that I have no doubt that sooner or later it will be possible to demonstrate such an assumption in an unambiguous and scientific way, or even repeat the process experimentally (Rolle, 1863).

In the book "The Systems View of Life: A Unifying Vision" (Capra \& Luisi, 2014) the authors integrate in a single framework of coherent thought the ideas, models and theories that are the foundation of the systemic vision of life, highlighting its the economic, ecological, political and spiritual implications.

Personally, I would like to reiterate and underscore some fundamental concepts in the book which I completely share.

Life is a network of complex and inseparable relationships that renders the understanding of an individual phenomenon indivisible from the understanding the entire ecosystem in which it occurs. Therefore the answers can not be found by relying exclusively to the scientific method. A "holistic" approach is now required, one that is able to reflect on connectivity, relationships and contexts as well as properties and functions of the individual parts.

The discipline that best reflects the systemic vision of life is ecology, which reconnects the life sciences with the earth sciences and studies the interaction of organisms with each other and with the surrounding environment. The new ecological science - that has emerged from organismic biology only in the late twentieth century, when the concept of ecosystem developed - is not anthropocentric but eco-centric, that is characterized by the awareness that all living things are tied together in networks of interdependence.

Ecology is the ideal bridge between science and spirituality. In fact, within the systemic view of life, it is essential the concept of balance between science - responsible for the material and 
technological progress - and spirituality, responsible for the internal growth of individuals and ethical limitations imposed by the excessive consumption of resources of the planet. The balance between science and spirituality determines the welfare of society.

The bridge between the Big Bang and Biology ferries us from the original point to the biologically active side where sentient life and then science start. But to close correctly the "run" of it, it is necessary to cross one bridge more: The bridge between science and spirituality. If this bridge is properly covered, our society will flourish.

Personally, I feel obligated to point out some observations that seem fundamental about the philosophical and social implications of contemporary science. These observations lead to interesting conclusions about the origin of life and self-organization of natural and synthetic systems. These findings are in keeping with the Buddhist view of the universe. It is understood as a living organism being composed of myriads of components all related and interacting with one another. Life can be seen as a system of interconnected autopoietic systems. The organism interacts with the environment in a "cognitive" way. At the same time, the organism "creates" its own environment and the environment allows the creation of the organism. But this is the concept of dependent origin. According to this concept, every phenomenon is the product of the interaction of every other phenomenon in the universe.

The consequence of this view are of extraordinary importance, above all in ethics: it asserts that all living beings and their environment are inextricably linked, and that their essence is not absolute but "of relationship." It leads us to respect every individual being and its inherent rights. In other words, this view leads us to live and act without distinguishing our own happiness from that of others. Ultimately this view leads to the TOTAL RESPECT OF LIFE in the most general meaning.

\section{Acknowledgements}

It is my pleasure to thank:

\section{- the Scientific Organizing Committee:}

- James H. Beall - NRL/SSD, Washington D.C. \& SJC, Annapolis, USA

- Dmitry Bisikalo - RAS - Institute of Astronomy, Moscow, Russian Federation

- Guennady Bisnovatyi-Kogan - RAS - Space Research Institute, Moscow, Russian Federation

- Riccardo Claudi - INAF-Osservatorio Astronomico di Padova, Italy

- Franco Giovannelli - INAF - Istituto di Astrofisica e Planetologia Spaziali, Roma, Italy (Chairperson)

- René Hudec - ASCR/AI, Ondrejov \& CTU, Prague, Czech Republic

- Pieter Meintjes - University of the Free State, Bloemfontein, South Africa

- Joanna Mikolajewska - PAN - N. Copernicus Astronomical Center, Warsaw, Poland 
- Lola Sabau-Graziati - INTA - Departamento de Cargas Utiles y Ciencias del Espacio, Madrid, Spain

- Andrea Santangelo - IAA - Universität Tübingen, Germany

- Giora Shaviv - Department of Physics, Technion, Haifa, Israel

- Edward Sion - Villanova University, Villanova, PA, USA

- Pietro Ubertini - INAF - Istituto di Astrofisica e Planetologia Spaziali, Roma, Italy

\section{- The super-efficient Local Organizing Committee:}

- Dmitry Bisikalo - RAS - Institute of Astronomy, Moscow, Russian Federation (Chairperson)

- Franco Giovannelli - INAF - Istituto di Astrofisica e Planetologia Spaziali, Roma, Italy (CoChairperson)

- Valery Shematovich - RAS - Institute of Astronomy, Moscow, Russian Federation (CoChairperson)

- Anna Fateeva - RAS - Institute of Astronomy, Moscow, Russian Federation

- Francesco Reale - CNR - Istituto dei Sistemi Complessi, Roma, Italy (Co-Chairperson)

- Polina Isakova - RAS - Institute of Astronomy, Moscow, Russian Federation

\section{- The Directors of sponsoring Institutions}

Special thanks to Francesco Reale, alias FIGARO, for his tireless efforts so that the workshop could take place perfectly. Even the role of perfect sommelier at the table of Anya Fateeva and Anna Lisa Amodio, as shown in Fig. 5.

Particular warm thanks to:

- ALL SPEAKERS, who rendered this workshop very interesting

- ALL PARTICIPANTS, who breathed life into the workshop with their question-answers, and for the friendly atmosphere they provided

- The FOUR COLLEAGUES and FRIENDS (Nikolai Shakura, Paul Mason, Dmitry Bisikalo, and Janusz Ziółkowski) who kindly accepted the not so easy task of making the concluding remarks of the workshop.

- Flavia Giovannelli and Anna Lisa Amodio for their Superb Performance PO Box 1663 Santa Fe (The wives of Los Alamos).

- The staff of the Hotel Ambassador.

But, as you probably suspected, this workshop has been organized under peaceful and friendly surroundings, as also shown in Fig. 6, representing one scene of the performance of Anna Lisa and Flavia. 


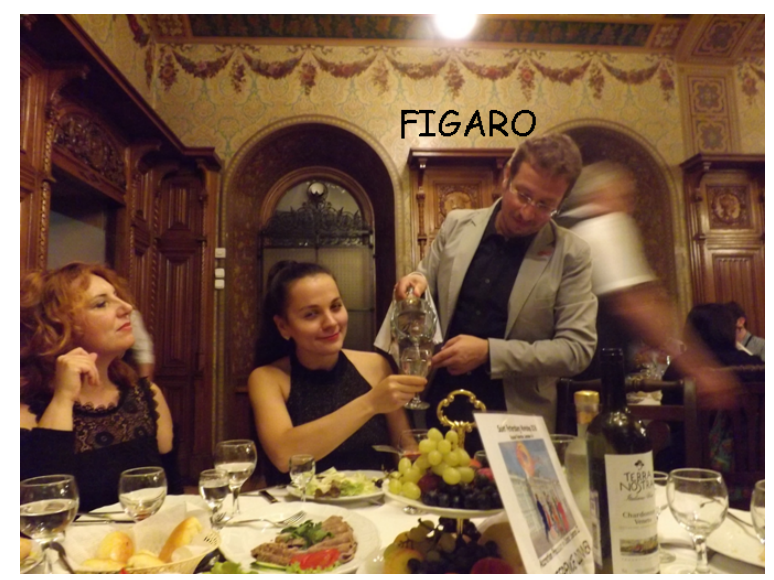

Figure 5: Francesco Reale, alias FIGARO, perfect sommelier at the table of Anna Lisa Amodio (left) and Anya Fateeva (right).

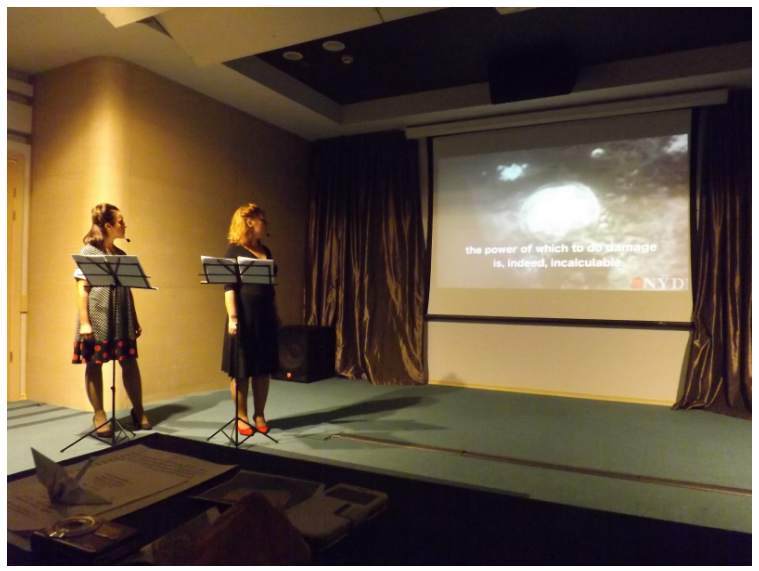

Figure 6: Flavia Giovannelli (left) and Anna Lisa Amodio (right) in a scene of their performance: $P O B O x$ 1663 Santa Fe (The wives of Los Alamos).

\section{Imagine}

Imagine all the people singing (Fig. 8). Imagine all the people dancing (Fig. 9).

You may say I'm a dreamer - But I'm not the only one - I hope someday you'll join us - And the world will live as one (John Lennon, 1971).

\section{Conclusions}

As I discussed in the introduction, I can state that the Universe is interconnected in all its components: from cosmic network, to clusters of galaxies, to galaxies, to stars, to planets, to living beings, up to the simple bacterium. Therefore every manifestation of life on our planet is subject to interconnection with all the surrounding environment. I can affirm that the whole Universe is a vital whole interconnected with more or less strong links between the various components, but that certainly exist. 


Imagine there's no heaven
It's easy if you try
No hell below us
Above us only sky
Imagine all the people living for today
Imagine there's no countries
It isn't hard to do
Nothing to kill or die for
And no religion too
Imagine all the people living life in peace
(John Lennon, 1971)

Figure 7: The words of the wonderful song Imagine by John Lennon (1971).
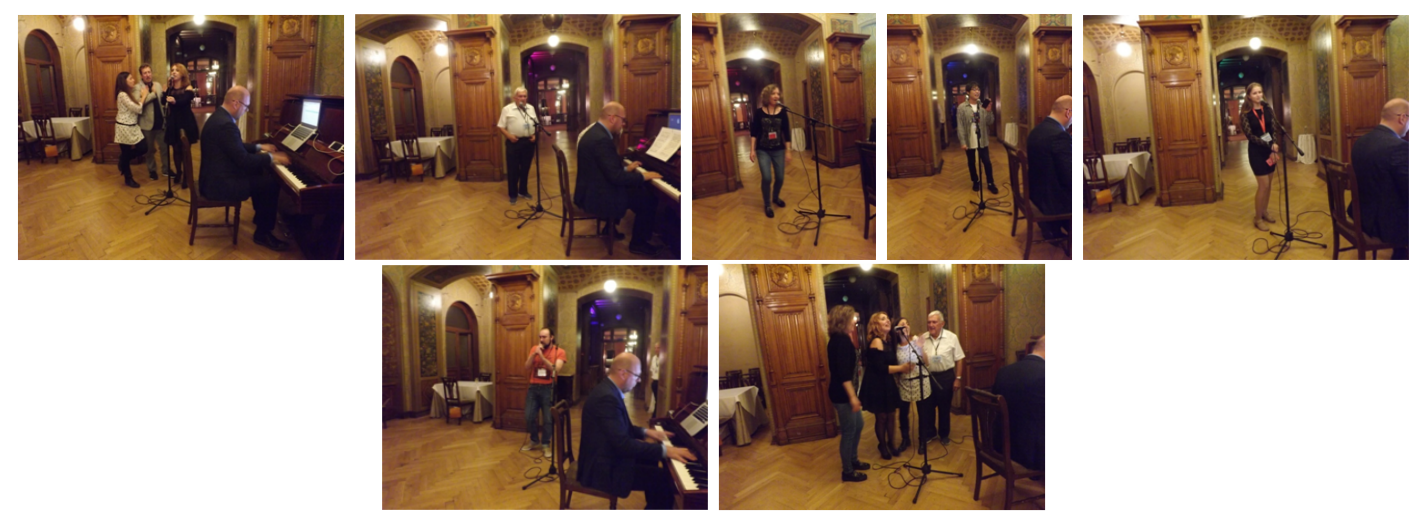

Figure 8: Amateur Singers after the Official Banquet.
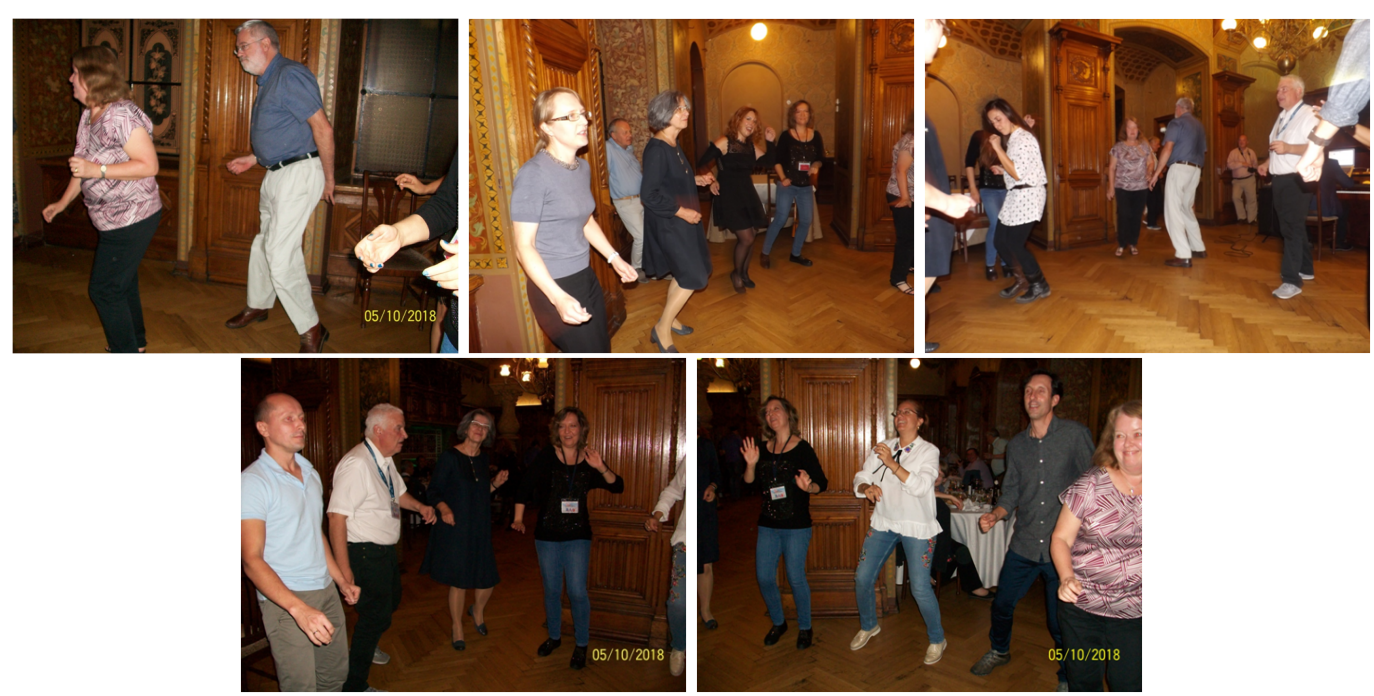

Figure 9: Amateur dancers after the Official Banquet. 
I have discussed about the accretion processes in cosmic sources of different nature. We have learned that the accretion is universal as shown in the cartoon of Fig. 10 (Jaime Sanchez Calleja, 2016).

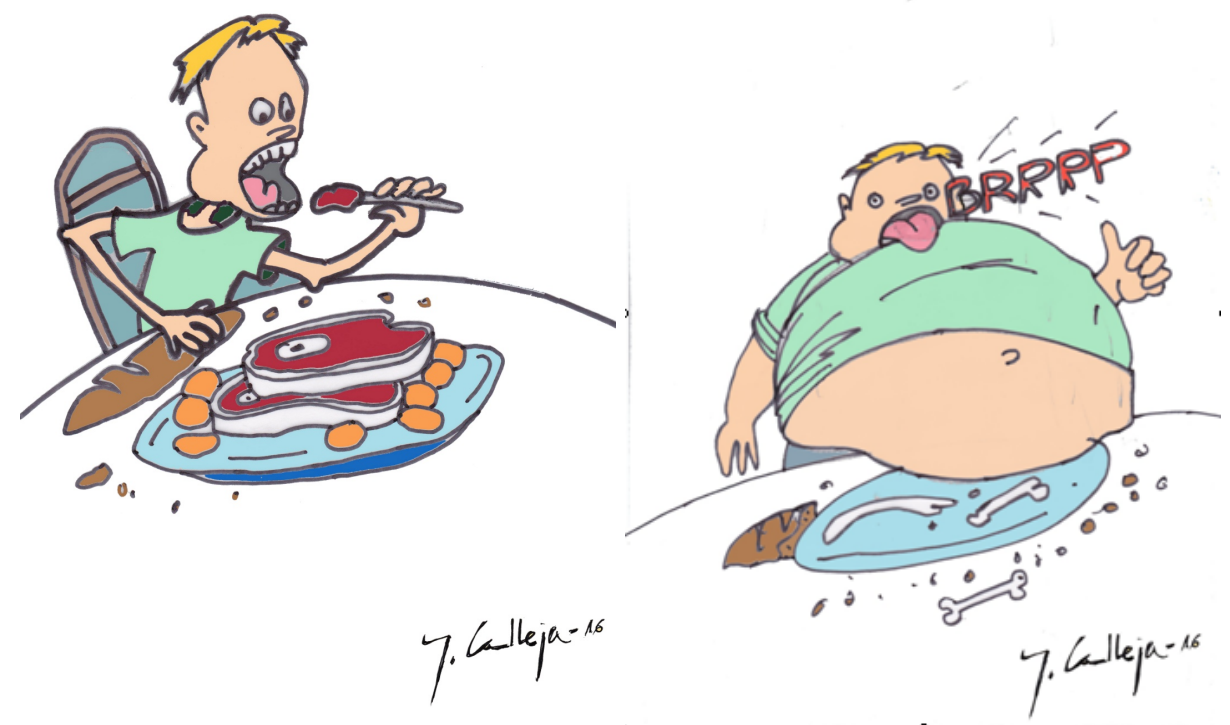

Figure 10: Left panel: Mass accretion onto a thin guy. Right panel: The fat guy after the accretion process (Jaime Sanchez Calleja, 2016).

Finally I can conclude with Fig. 11 that clearly explain all the mysteries of our Universe (Giovannelli, 2000). People who are able to read this sentence can understand that "The truth is written in the book of the Nature. We must learn to read this book".

$$
\begin{aligned}
& \text { 自然以山教科書には } \\
& \text { 真央がある。 } \\
& \text { ただ我々は } \\
& \text { 読み方を学ば } \\
& \text { なければならない }
\end{aligned}
$$

Figure 11: Understanding our Universe (Giovannelli, 2000).

The experiments provide the basic alphabet, immersed in an apparently chaotic soup, but necessary to understand the nature. From that soup we must extract words and phrases to compose 
the book of the nature. In other words, the data coming from the experiments constitute the basic alphabet that we use for constructing models that attempt to describe the nature. But we have a lot of models for interpreting the experimental data by the light of science. Depending on the hypotheses the results could run against the experiments. Then, in order to be acceptable, models can take into account and justify ALL the available data.

The same concept was expressed in much more incisive terms by Richard Phillips Feynman - Nobel laureate in Physics in 1965 - also known as The Great Explainer: It doesn't matter how beautiful your theory is, it doesn't matter how smart you are. If it doesn't agree with experiment, it's wrong.

\section{I hope to meet all of you once again during our next Saint Petersburg Workshop.}

Acknowledgments This research has made use of:

- The NASA's Astrophysics Data System;

- the NASA Exoplanet Archive, which is operated by the California Institute of Technology, under contract with the National Aeronautics and Space Administration under the Exoplanet Exploration Program;

- the Exoplanet Orbit Database and the Exoplanet Data Explorer at exoplanets.org.

\section{References}

[1] ALMA Science Advisory Committee: 2015, Major Science Themes in the 2020-2030 Decade, https://science.nrao.edu/facilities/alma/alma-develop-old-022217/ScienceThemes.pdf

[2] Antoniucci, S.: 2018, talk at the Saint Petersburg Workshop on Accretion Processes in Cosmic Sources - II.

[3] Boller, T.: 2017, talk at the Frascati Workshop 2017 on Multifrequency Behaviour of High Energy Cosmic Sources - XII.

[4] Bonfils, X., Delfosse, X., Udry, S., Forveille, T., Mayor, M. et al.: 2013. A\&A 549, id.A109, 75 pp.

[5] Bruno Giordano Nolano: 1584, De l'infinito, universo e mondi, Stampato in Venezia, Anno MDLXXXIV, in Dialoghi filosofici italiani, a cura di Michele Ciliberto, Mondadori, Milano (2000).

[6] Capra, F. \& Luisi, P.L.: 2014, The Systems View of Life: A Unifying Vision, Cambridge University Press.

[7] Carpenter, J., Iono, D., Testi, L., Whyborn, N., Wootten, A., Evans, N. (The ALMA Development Working Group): 2018, The Alma Development Roadmap, https://www.almaobservatory.org/en/publications/the-alma-development-roadmap/

[8] Cleeves, L. Ilsedore, Bergin, Edwin A., Alexander, Conel M. O.'D., Du, Fujun, Graninger, Dawn et al: 2014, Science $345,1590-1593$.

[9] Drake, F.D.: 1962, Intelligent Life in Space, New York: Macmillan, 128 pp. 
[10] Giovannelli, F. (Ed.): 1985, Multifrequency Behaviour of Galactic Accreting Sources, SIDEREA, Roma, Italy, pp. 371.

[11] Giovannelli, F.: 2000, in The Evolution of The Milky Way, F. Matteucci \& F. Giovannelli (Eds.), Kluwer Academic Publishers, pp. 619-620.

[12] Giovannelli, F.: 2016, in Accretion Processes in Cosmic Sources (APCS2016), Online at http://pos.sissa.it/cgi-bin/reader/conf.cgi?confid=288, id.65

[13] Giovannelli, F., Sabau-Graziati, L.: 2018, in Frontier Research in Astrophysics - III, https://pos.sissa.it/cgi-bin/chairman/chlist.cgi?confid=331, in press.

[14] Ikeda, D.: 2001a, For Today and Tomorrow - The thought of 30th May, Edizioni Esperia, Italy.

[15] Ikeda, D.: 2001b, For Today and Tomorrow - The thought of 11th June, Edizioni Esperia, Italy.

[16] Lennon, John: 1971, Lyrics (C)EMI Music Publishing. Lyrics powered by LyricFind.

[17] Lineweaver, C.H., Chopra, A.: 2012, Ann. Rev. of Earth and Planetary Sci., 40, issue 1, 597-623.

[18] Luisi, P.L.: 2006, The Emergence of Life. From Chemical Origin to Synthetic Biology, Cambridge Univeristy Press.

[19] Martino, B.L.: 2018, Very funny cartoons produced in occasion of the 2018 Saint Petersburg Workshop on Accretion Processes in Cosmic Sources - II, Saint Petersburg (Russian Federation, 3-8 September).

[20] Maruyama, S., Ebisuzaki, T., Kurokawa, K.: 2018, talk at the Mondello Workshop on Frontier Research in Astrophysics - II, Palermo, Italy, May 28 - June 2.

[21] Rixner, T.A., Siber, T.: 1824, Leben und Lehrmeinungen berühmter Physiker, Sulzbach, Heft 5.

[22] Rolle, F.: 1863, Ch.Darwin's Lehre von der Entstehung der Arten, in J.C. Hermann, Ihrer Anwendung auf die Schöpfunggeschichte.

[23] de Saint Exupéry, Antoine: 1943, Le Petit Prince, Gallimard, France. English version The Little Prince, Reynal \& Hitchcock, U.S.A.

[24] Salahuddin Armstrong, P.: 2014, April 13, Speech originally delivered at the New Humanity Movement event.

[25] Sanchez Calleja, J.: 2016, Very funny cartoons produced in occasion of the 2016 Saint Petersburg Workshop on Accretion Processes in Cosmic Sources, Saint Petersburg (Russian Federation, 5-10 September). 